\title{
Two consecutive days of treatment with liposomal cisplatin in non-small cell lung cancer
}

\author{
G.P. STATHOPOULOS ${ }^{1}$, J. STATHOPOULOS ${ }^{1}$ and J. DIMITROULIS ${ }^{2}$ \\ ${ }^{1}$ First Oncology Clinic, Errikos Dunant Hospital; ${ }^{2} 6$ th Pulmonary Clinic, Hospital of Thoracic Diseases, Athens, Greece
}

Received January 15, 2012; Accepted May 16, 2012

DOI: $10.3892 / \mathrm{ol} .2012 .836$

\begin{abstract}
Liposomal cisplatin (Lipoplatin) is a new agent, a cisplatin formulation that has been investigated in a number of studies and compared with cisplatin with respect to toxicity and effectiveness. It has been administered once weekly and in combination with a second agent, once every two weeks. The main outcome of the studies was that lipoplatin has no renal toxicity and is as equally effective as cisplatin. The present study investigated toxicity and effectiveness when lipoplatin is administered on two consecutive days, repeated every two weeks. Between January 2011 and November 2011, a total of 21 patients with histologically- or cytologically-confirmed non-small cell lung cancer (NSCLC) were enrolled in the study. All but two patients, who had not been pretreated, had received one or two series of chemotherapy and some had undergone radiotherapy. Lipoplatin monotherapy was infused for $8 \mathrm{~h}$ the first and second days and repeated every 2 weeks with the aim of administering 6 cycles. The dose per day was $200 \mathrm{mg} / \mathrm{m}^{2}$. Eight out of 21 (38.10\%) patients had a partial response, $9(42.86 \%)$ had stable disease and 4 $(19.05 \%)$ had progressive disease. Results showed that there was no renal failure toxicity and no other adverse reactions apart from grade 1 myelotoxicity in only 2 patients who had been heavily pretreated, and grade 1 nausea/vomiting in 4 patients. Liposomal cisplatin is an agent with negligible toxicity and reasonably high effectiveness even when administered to pretreated patients with NSCLC.
\end{abstract}

\section{Introduction}

The new agent, liposomal cisplatin (Lipoplatin), has been investigated in pre-clinical and clinical studies in recent years, and as yet there are more than 16 reports published in peer-reviewed journals (1). This agent was produced as a substitute for cisplatin and it has resulted in a reduction in toxicity compared to cisplatin, but with equal effectiveness.

Correspondence to: Dr G.P. Stathopoulos, First Oncology Clinic, Errikos Dunant Hospital, Semitelou 2A, 11528 Athens, Greece E-mail: dr-gps@ath.forthnet.gr

Key words: liposomal cisplatin, lipoplatin, toxicity, effectiveness, non-small cell lung cancer
Cisplatin has been in use for over 30 years and has been demonstrated to be an effective agent against a number of malignancies, including lung, ovarian, head and neck, gynaecological, testicular and urothelial cancers (2-10).

Although cisplatin is one of the most significant and effective anticancer agents, its toxicity is often an inhibiting factor preventing the continuation of treatment courses. The main side effect is renal toxicity (renal failure). Other adverse reactions have included nausea and vomiting, asthenia and neurotoxicity (11-14).

Over the last 15-20 years, there has been an extensive effort to produce other agents as a substitute for cisplatin. The main substitutive agent was the CDDP analogue, carboplatin. Moreover, in certain malignancies other new agents, including taxanes (paclitaxel, docetaxel) and gemcitabine and vinorelbine, have been tested. Renal toxicity was avoided with the use of these agents, but other side effects, including myelotoxicity, were observed. However, none of these agents were more effective when compared with cisplatin (15-21).

Liposomal cisplatin has been tested in patients with pancreatic, breast and mainly non-small cell lung cancer (NSCLC). The lipids of lipoplatin are composed of soy phosphatidyl choline (SPC-3), cholesterol, dipalmitoyl phosphatidyl glycerol (DPPG) and methoxy-polyethylene glycol-disteroyl phosphatidyl ethanolamine. The formulation was achieved by the formation of reverse micelles between cisplatin and DPPG under special conditions of $\mathrm{pH}$, ethanol, ionic strength and other parameters. Lipoplatin has demonstrated a high increase of concentration in primary or metastatic tumors, with levels up to 10 to 50-fold higher than the uptake of the normal tissue adjacent to the tumor (22). Despite the number of publications related to lipoplatin, an analytical study evaluating the value of this agent with respect to toxicity and the modified two days of treatment is required.

Thus, the aim of the present study of lipoplatin, knowing its negligible toxicity, was to infuse this agent as monotherapy on days 1 and 2 every 2 weeks in pretreated and non-pretreated patients with NSCLC, and to determine the effectiveness of this treatment modification and whether toxicity is increased.

\section{Materials and methods}

Lipoplatin method of administration. The agent was infused for $8 \mathrm{~h}$; the duration of time which has been established by other studies $(1,22)$. As yet, no serious toxicity has been 
determined when lipoplatin is administered as monotherapy or in combination with another agent (23).

To date, lipoplating has been administered once every week with no increase in side effects, while it is rarely administered once every 3 weeks. It has also been administered on days 1 and 8 and repeated on the 21 st day (24-28). To determine the toxicity and effectiveness in the present study, the agent was administered for 2 consecutive days every 2 weeks.

\section{Eligibility criteria}

Patients. Patients aged $>18$ years with a histologically- or cytologically-confirmed diagnosis of NSCLC stage IV with bidimensionally measurable disease were enrolled in the study. Two patients had not undergone prior chemotherapy or radiotherapy, while the remaining had pre-treatment of first- or second-line chemotherapy. Other eligibility criteria included a World Health Organisation (WHO) performance status (PS) of 0-2, a life expectancy of at least 3 months, adequate bone marrow reserve (granulocyte count, $1500 \mu 1$ ${ }^{1}$; platelet count, $120000 / \mu \mathrm{l}^{-1}$ ), normal renal function (serum creatinine concentration, $<1.5 \mathrm{mg} / \mathrm{dl}$ ) and liver function tests (total serum bilirubin, $<3 \mathrm{mg} / \mathrm{dl}$; provided that serum transaminases and serum proteins were normal), and normal cardiac function with no history of clinically unstable angina pectoris or myocardial infarction or congestive heart failure within the 6 months prior to the study. Patients with central nervous system involvement were eligible if they were asymptomatic. Patients with active infection, malnutrition or a second primary tumor (with the except of a non-melanoma skin epithelioma or in situ cervix carcinoma) were excluded from the study. The study was approved by our institutional review boards and all patients provided written informed consent to participate.

Treatment plan. Patients were treated on an outpatient basis. Lipoplatin was administered on days 1 and 2, and every 2 weeks again for two days. The treatment was designed to administer 6 courses at minimum (each course involved the two consecutive days of administration). The dose was $200 \mathrm{mg} /$ $\mathrm{m}^{2}$ per day based on the maximum tolerated dose defined by a previous phase I study (23). Lipoplatin was produced by Regulon Inc. (Mountain View, CA, USA) and Regulon AE (Alimos, Athens, Greece).

The Lipoplatin infusion time was $8 \mathrm{~h}$. According to pharmacokinetics, there is slow renal excretion whereby $40 \%$ of the drug is excreted in 3 days (29). Premedication involved $8 \mathrm{mg}$ of ondansetron and $8 \mathrm{mg}$ of dexamethasone. In cases of severe myelotoxicity, the treatment would have been postponed for 3-7 days. Toxicities were graded according to the WHO guidelines (30).

Patient evaluation. Pretreatment evaluation included complete medical history and physical examination, full blood count, including differential leukocyte and platelet counts, a standard biochemical profile (and creatinine clearance when necessary), electrocardiogram, chest X-ray, ultrasound of the upper abdomen and computed tomography (CT) scans of the chest, upper and lower abdomen. Additional imaging studies were performed upon clinical indication. Full blood counts were performed weekly. In cases of grade 3 and 4 neutropenia or thrombocytopenia, full blood counts were evaluated daily.

A detailed medical and physical examination was completed prior to each course. Biochemical tests, ECG and chest X-rays were performed every 4 weeks and CT scans were performed at the end of the 3 rd cycle.

Definition of response. For the assessment of response, we used imaging-based evaluation. A complete response (CR) was considered to be the disappearance of all measurable disease confirmed at 6 weeks at the earliest. Partial response (PR) was a $30 \%$ tumor decrease, while stable disease (SD) was determined if neither the PR nor the progressive disease (PD) criteria were met; indicating a $20 \%$ increase in tumor burden in PD, but not for CR, PR or SD documented before increased disease. Response data were based on the response evaluation criteria in solid tumors (RECIST) (31). A two-step deterioration in performance status (PS), a $>10 \%$ loss in pretreatment weight or increasing symptoms, did not constitute progression of the disease. However, the progression of these complaints was followed by a new evaluation of the extent of the disease. All responses had to be maintained for at least 6 weeks and be confirmed by an independent panel of radiologists.

Statistical analysis. Simon's two-stage minimax design was used for the calculation of the sample size. The significance level was set at $5 \%$ and the power at $90 \%$. The low response probability was set at $20 \%$ and the level of useful activity at $40 \%$. In the first stage, 15 patients were enrolled in the study. If at least five responses were observed, more patients were recruited. For the main objective, which was to determine the toxicity, 20 patients were considered to be sufficient.

The primary endpoints of the study were to determine the toxicity (adverse reactions) and tumor responsiveness. The duration of the response was calculated from the day of the first demonstration of response until PD. Overall survival (OS) was calculated from the day of enrollment until the end of the study or death. Time to tumor progression was calculated from day of entry into the study until documented PD. The estimation of survival distribution was calculated by the Kaplan-Meier method.

\section{Results}

Patient characteristics. A total of 21 patients were recruited into the study between January 2011 and November 2011. According to the statistical design, this number of patients was considered adequate with respect to the objective of the study.

The 21 patients comprised 20 males and 1 female (Table I). Of the 21 patients, 19 patients had adenocarcinoma and 2 had squamous cell carcinoma. The majority of patients had low differentiation disease. Metastasis was observed in the liver, bones, other lung, adrenal gland and brain in 3 patients (the latter had undergone radiation therapy).

Compliance with treatment. Seventy-five cycles were administered in total (150 infusion days). The median number of cycles was 4 and the range was 1-6. No patient had treatment delay due to myelotoxicity or other side effects; only 2 patients 
Table I. Characteristics of the 21 patients included in the study.

\begin{tabular}{|c|c|c|}
\hline & No. of patients & $\%$ \\
\hline Patients enrolled & 21 & 100 \\
\hline Patients evaluable & 21 & 100 \\
\hline \multicolumn{3}{|l|}{ Gender } \\
\hline Male & 20 & 95.24 \\
\hline Female & 1 & 4.76 \\
\hline \multicolumn{3}{|l|}{ Age (years) } \\
\hline Median & 64 & \\
\hline Range & $38-76$ & \\
\hline \multicolumn{3}{|l|}{ Disease stage } \\
\hline IIIA & 0 & 0 \\
\hline IIIB & 0 & 0 \\
\hline IV & 21 & 100 \\
\hline \multicolumn{3}{|l|}{ Histology } \\
\hline Adenocarcinoma & 19 & 90.48 \\
\hline Squamous cell carcinoma & 2 & 9.52 \\
\hline \multicolumn{3}{|l|}{ Performance status } \\
\hline 0 & 6 & 28.57 \\
\hline 1 & 7 & 33.33 \\
\hline 2 & 8 & 38.10 \\
\hline
\end{tabular}

had a one-week delay due to a respiratory infection which was treated with antibiotics. Drug dose reduction was not required due to adverse reactions and no growth factor was administered. At the time of analysis, 2 patients had received the treatment as first-line, 10 as second-line and 9 as third-line. Nine patients remained alive and well at the end of the study, 9 patients succumbed to the disease, 2 patients succumbed to a heart attack 2 months after the end of the treatment and 1 patient was lost to follow-up.

Response rate and survival. Survival was evaluated on an intention-to-treat basis. There was no CR in the 21 evaluable patients. Eight (38.10\%) patients achieved a PR, $9(42.86 \%)$ had SD and 4 (19.05\%) had PD (Table II). Among the responders, 2 patients underwent first-line treatment, 5 second-line and 1 patient had third-line treatment. Four patients with SD had second-line treatment and 5 had third-line. Among the nonresponders (PD), 1 patient had second-line treatment and 3 patients had third-line. No PD was observed in any of the patients who achieved a PR for 4-6 months after treatments, and the median time of survival of the 8 patients with a PR was 7 months, range $3-10+$ months. It is worth mentioning that in two patients with a minor response the tumor biopsy examination after treatment was full of necrotic cells.

Toxicity. All 21 patients were evaluable for toxicity. There was no myelotoxicity (neutropenia, thrombocytopenia or anemia) in 19 of the 21 patients. Two (9.52\%) patients experienced grade 1 myelotoxicity, but these patients had been heavily pretreated. Grade 1 nausea and vomiting on the first or second day after treatment was observed in $4(19.05 \%)$ patients. Grade 1 fatigue and peripheral neuropathy were observed in
Table II. Response rates.

\begin{tabular}{lccc}
\hline & $\begin{array}{c}\text { 2-day treatment of lipoplatin } \\
\text { every 2 weeks }\end{array}$ & No. of patients & $\%$ \\
\hline CR & & 0 & 0 \\
PR & 1st line & 2 & 38.10 \\
& 2nd line & 5 & \\
SD & 3rd line & 1 & \\
& 2nd line & 4 & 42.86 \\
PD & 3rd line & 5 & \\
& 2nd line & 1 & 19.05 \\
& 3rd line & 3 & \\
\end{tabular}

$\mathrm{CR}$, complete response; $\mathrm{PR}$, partial response; $\mathrm{SD}$, stable disease; $\mathrm{PD}$, progressed disease.

$3(14.29 \%)$ patients. No alopecia was observed. During the time of the drug infusion, temporary myalgia was observed in 5 patients, but it lasted for only 5-10 min. Notably, no renal toxicity (blood urea-serum creatinine were not increased) was detected, even after the 6th treatment course.

\section{Discussion}

This study presents a new type of liposomal administration, with the infusion of the drug on days 1 and 2, with repetition every 2 weeks. It was determined that this agent can easily be administered for two consecutive days without causing serious adverse reactions, and particularly without causing renal toxicity. The results showed that patients were able to tolerate 4 lipoplatin infusions in 2 weeks. The determination in this study of the negligible toxicity of lipoplatin indicates that it may be administered even as first-line treatment to patients with NSCLC who would not be able to tolerate the serious adverse reactions caused by other agents. Patients with lung cancer who may have renal insufficiency, cardiac problems or other chronic disease could be selected for this modified two consecutive days of treatment every 2 weeks. The results of the present study and those of another study presented at the 2011 ASCO Congress may provide enough data concerning the choice of treatment for patients with NSCLC (32). If lipoplatin is combined with another agent, such as paclitaxel, vinorelbine or gemcitabine, there is no requirement for lipoplatin dose reduction.

The value of liposomal cisplatin in clinical practice, mainly in patients with NSCLC, may gradually establish it as a substitute for cisplatin. In this study, the effectiveness of lipoplatin was reasonably high, even in pretreated patients with NSCLC.

In the present study, a two-day treatment of liposomal cisplatin has been investigated and negligible toxicity determined. Renal, myelotoxicity (apart from grade 1) and other side effects were not observed, even with the administration of the drug at the maximum tolerated dose on the first and second days. Effectiveness remained high even in pretreated patients with NSCLC. 


\section{References}

1. Stathopoulos GP and Boulikas T: Lipoplatin formulation review article. J Drug Deliv: Aug 29, 2011 (Epub ahead of print).

2. Non-Small-Cell Lung Cancer Collaborative Group: Chemotherapy in non-small-cell lung cancer, a meta-analysis using updated data on individual patients from 52 randomised clinical trials. BMJ 311: 899-909, 1995.

3. Shepherd FA, Dancey J, Arnold A, et al: Phase II study of pemetrexed disodium, a multitargeted antifolate and cisplatin as first line therapy in patients with advanced non-small-cell lung cancer. Cancer 92: 595-600, 2001.

4. Ardizzoni A, Boni L, Tisseo M, et al: Cisplatin versus carboplatin-based chemotherapy in first line treatment of advanced non-small-cell lung cancer: an individual patient data metaanalysis. J Natl Cancer Inst 99: 847-857, 2007.

5. Aabo K, Adams M, Adnitt P, et al: Chemotherapy in advanced ovarian cancer: four systemic meta-analysis of individual patient data from 37 randomized trials. Br J Cancer 78: 1479-1487, 1998.

6. Taylor AE, Wiltsaw E, Gore ME, et al: Long-term follow-up of the first randomized study of cisplatin versus carboplatin for advanced epithelial ovarian cancer. J Clin Oncol 12: 2066-2070, 1994.

7. Pignon JP, Bourhis J, Domange C and Designé L: Chemotherapy added to locoregional treatment for head and neck squamous cell carcinoma: three meta-analyses of updated individual data. Lancet 355: 949-955, 2000.

8. Einform LH, Williams SD, Loehrer PT, et al: Evaluation of optimal duration of chemotherapy in favourable-prognosis disseminated germ cell tumors: a Southeastern Cancer Study Group Protocol. J Clin Oncol 7: 387-391, 1989.

9. Kaufman D, Raghavan D, Carducci M, et al: Phase II trial of gemcitabine plus cisplatin in patients with metastatic urothelial cancer. J Clin Oncol 18: 1921-1927, 2000.

10. Rosenberg B: Platinum complexes for the treatment of cancer: why the research goes on. In: Cisplatin: Chemistry and Biochemistry of a Leading Anticancer Drug. Lippert B (ed). Verlag Helvetica Chemica Acta, Zurich, pp3-12, 1999.

11. Hayes DM, Critkovic E, Golbey RB, et al: High dose cis-platinum diammine dichloride: amelioration of renal toxicity by mannito diuresis. Cancer 39: 1372-1381, 1977.

12. Sorenson CM and Eastman A: Mechanism of cis-diamminedichloroplatinum (II)-induced cytotoxicity: role of G2 arrest and DNA double-strand breaks. Cancer Res 48: 4484-4488, 1988.

13. Gandara DR, Nahhas NA, Adelson MD, et al: Randomized placebo-controlled multicenter evaluation of diethyldithiocarbamate for chemoprotection against cisplatinum-induced toxicities. J Clin Oncol 13: 490-496, 1995.

14. Arany I and Safirstein RL: Cisplatin nephrotoxicity. Semin Nephrol 23: 460-464, 2003.

15. Tognoni A, Pensa F, Vaira F, et al: A dose finding study of carboplatin and gemcitabine in advanced non-small-cell lung cancer. J Chemother 14: 296-300, 2002.

16. Johnson BE: Integration of new agents into the treatment of advanced non-small-cell lung cancer. In: American Society of Clinical Oncology Educational Book. Perry MC, Govindan R (eds). Lipincott Williams and Wilkins, Baltimore, pp345-356, 2000 .

17. Stathopoulos GP, Veslemes M, Georgatou N, et al: Paclitaxel and vinorelbine combination in advanced inoperable adenocarcinoma of the lung: a phase II study. Anticancer Res 23: 3479-3484, 2003.
18. Stathopoulos GP, Dimitroulis J, Antoniou D, et al: Front-line paclitaxel and irinotecan combination chemotherapy in advanced non-small-cell lung cancer: a phase I-II trial. Br J Cancer 93: 1106-1111, 2005.

19. Fosella FV, DeVore R, Kerr RN, et al: Randomized phase III trial of docetaxel versus vinorelbine or ifosfamide in patients with advanced non-small-cell lung cancer previously treated with platinum-containing chemotherapy regimens. J Clin Oncol 18: 2354-2362, 2000.

20. Stathopoulos GP, Veslemes M, Georgatou N, et al: Front-line paclitaxel-vinorelbine versus paclitaxel-carboplatin in patients with advanced non-small-cell lung cancer: a randomized phase III trial. Ann Oncol 15: 1048-1055, 2004.

21. Comella P, Fresci G, Panza N, et al: Randomized trial comparing cisplatin, gemcitabine and vinorelbine with either cisplatin and gemcitabine or cisplatin and vinorelbine in advanced non-small cell lung cancer: interim analysis of a phase III trial of the Southern Italy Cooperative Oncology Group. J Clin Oncol 18: 1451-1457, 2000.

22. Boulikas T, Stathopoulos GP, Volakakis N and Vougiouka M: Systemic lipoplatin infusion results in preferential tumor uptake in human studies. Anticancer Res 25: 3031-3039, 2005.

23. Stathopoulos GP, Rigatos SK and Stathopoulos J: Liposomal cisplatin dose escalation for determining the maximum tolerated dose and dose-limiting toxicity: a phase I study. Anticancer Res 30: 1317-1321, 2010.

24. Stathopoulos GP, Boulikas T, Vougiouka M, et al: Liposomal cisplatin combined with gemcitabine in pretreated advanced pancreatic cancer patients: a phase I-II study. Oncol Rep 15: 1201-1204, 2005.

25. Koukourakis M, Giatromanolaki A, Pitakoudis M, et al: Concurrent liposomal cisplatin (lipoplatin), 5-fluorouracil and radiotherapy for the treatment of locally advanced gastric cancer: a phase I-II study. Int J Radiol Oncol Biol Phys 78: $150-155,2009$.

26. Karpathiou G, Argiana E, Koutsopoulos A and Froudarakis ME: Response of a patient with pleural and peritoneal mesothelioma after second-line chemotherapy with lipoplatin and gemcitabine. Oncology 73: 426-429, 2008.

27. Farhat FS, Ibrahim K, Kattan J, et al: Preliminary results of Phase II study of liposomal cisplatin-vinorelbine combination as first-line treatment in HER2/neu negative metastatic breast cancer (MBC). Proceedings of the ASCO Annual Meeting, abs. 31351, 2009

28. Mylonakis M, Athanasiou A, Ziras N, et al: Phase II study of liposomal cisplatin (lipoplatin) plus gemcitabine versus cisplatin plus gemcitabine as first line treatment in inoperable (stage IIIB/IV) non-small-cell lung cancer. Lung Cancer 68: 240-247, 2010.

29. Stathopoulos GP, Boulikas T, Vougiouka M, et al: Pharmacokinetics and adverse reactions of a new liposomal cisplatin (lipoplatin): phase I study. Oncol Rep 13: 589-595, 2005.

30. Miller AB, Hoogstraten B and Stagnet M: Reporting results of cancer treatment. Cancer 47: 207-214, 1981.

31. Therasse P, Arbuck SG, Eisenhower EA, et al: New guidelines to evaluate the response to treatment in solid tumors. European Organization for Research and Treatment of Cancer, National Cancer Institute of the United States, National Cancer Institute of Canada. J Natl Cancer Inst 92: 205-216, 2000.

32. Stathopoulos GP, Rigatos S, Stathopoulos J and Batzios S: Liposomal cisplatin in cancer patients with renal failure. Proceedings of the ASCO Annual Meeting. abs. 7072, 2011. 